\title{
Validation of the brief international cognitive assessment for multiple sclerosis (BICAMS) in the Portuguese population with multiple sclerosis
}

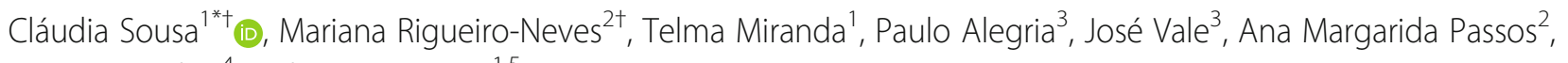
Dawn Langdon ${ }^{4}$ and Maria José Sá ${ }^{1,5}$

\begin{abstract}
Background: The validation of international cognitive batteries in different multiple sclerosis (MS) populations is essential. Our objective was to obtain normative data for the Portuguese population of the Brief International Cognitive Assessment for Multiple Sclerosis (BICAMS) and assess its reliability.

Methods: The BICAMS was applied to 105 MS patients and 60 age, gender and education matched healthy controls (HC). In order to test its reliability, BICAMS was re-administered in a subset of 25 patients after a 7-month interval.

Results: Most participants were women, with a mean age of 37, 21 years and a mean of 14,08 years of education. The vast majority of the MS patients (92.4\%) had the relapsing remitting type, $58.1 \%$ were professionally active, mean disease duration was 6.52 years, median EDSS score was 1.5 (range: 0-6.0) and the median MSSS score was 2.01 (IQR range: 3.83). The MS group presented significantly higher scores of anxiety and depression than $\mathrm{HC}$ and $47,4 \%$ had fatigue. The MS group performed significantly worse than the control group across the three neuropsychological tests, yielding the following values: SDMT: $t(165)=3.77, p=.000 ;$ CVLT-II: $t(165)=2.98, p=.003$; and BVMT-R: $t(165)=2.94, p=.004$. The mean raw scores for Portuguese normative data were as follows: SDMT: $58.68 \pm 10.02$; CVLT-II: $60.47 \pm 10.12$; and BVMT-R: $24.68 \pm 5.52$. Finally, test-retest reliability coefficients for each test were as follows: SDMT: $r=.90$; CVLT-II: $r=.71$; and BVMT-R: $r=.84$.
\end{abstract}

Conclusions: The Portuguese version of BICAMS here in described is a reliable monitoring instrument for identifying MS patients with cognitive impairment.

Keywords: Multiple sclerosis, Cognitive impairment, BICAMS, Normative values for Portugal

\section{Background}

Multiple sclerosis (MS) is a chronic inflammatory demyelinating disease of the central nervous system that can impair any body function, including cognition [1]. Cognitive dysfunction affects 40 to $70 \%$ patients $[2,3]$. Irrespective of age and gender [3, 4], may occur at all stages of the disease, even at the very early beginning $[5$,

\footnotetext{
* Correspondence: claudia-sousa@sapo.pt

†Cláudia Sousa and Mariana Rigueiro-Neves contributed equally to this work.

'MS Clinic, Department of Neurology, Centro Hospitalar São João Porto,

Alameda Prof. Hernâni Monteiro, 4200 - 319 Porto, Portugal

Full list of author information is available at the end of the article
}

6] and definitely impacts the lives of MS patients and their families $[3,7,8]$.

The characteristic pattern of cognitive impairment in MS has been described early on to include memory, information processing efficiency, executive functioning, attention and processing speed [1]. However, the cognitive domains most likely to be affected in MS are information processing speed and memory, whilst visual processing and executive function are less likely to be impaired and language is largely intact [1,9-12].

The most frequently used neuropsychological batteries for patients with MS such us, the Brief Repeatable Battery of Neuropsychological tests and the Minimal

(C) The Author(s). 2018 Open Access This article is distributed under the terms of the Creative Commons Attribution 4.0 International License (http://creativecommons.org/licenses/by/4.0/), which permits unrestricted use, distribution, and 
Assessment of Cognitive Function in MS, require specialized technical and human resources and take a considerable time for evaluation in the daily clinical setting $[1,13]$. Recently, the Brief International Cognitive Assessment for MS (BICAMS) was developed and recommended as a validated and standardized international screening test, because it is an easier assessment tool that can be administered by a technician who is not a specialist in neuropsychology and lasts only about 15 min to apply $[14,15]$. Besides, the three instruments that compose BICAMS - Symbol Digit Modalities Test (SDMT) [16], California Verbal Learning Test (CVLT-II) [17] and Brief Visuo-spatial Memory Test Revised (BVMT-R) [18] - have previously been shown to have good psychometric properties.

The aims of this study are to describe the normative values of the Portuguese version of the BICAMS with gender, age and education corrections and to test the validity of this battery in a sample of Portuguese patients with MS.

\section{Methods \\ Participants}

A group of 105 patients with MS diagnosed according to the McDonald criteria [19] and a control group of 60 age, gender and education matched healthy subjects (HC), entered this study, and conducted in the period 2015-2016.

The MS patients were consecutively recruited at the MS Clinics from two hospitals located in separate regions of the country, Hospital de São João (Oporto; North) and Hospital Beatriz Ângelo (Loures; South), whereas the HC group was recruited from the community and among relatives and friends of MS patients. All participants were aged between 17 and 69 years and they were fluent in Portuguese as first language.

Exclusion criteria were current or past neurological disorder other than MS, presence of major psychiatric illness, history of learning disability, history of serious head trauma, presence of alcohol or drug abuse, relapse and/or corticosteroid use within 4 weeks preceding the neuropsychological assessment. $\mathrm{HC}$ were also required to present scores $>21$ on Montreal Cognitive Assessment Portuguese version (MoCA) [20, 21].

The study was approved by the ethical committees of both hospitals. All the participants, from MS group and $\mathrm{HC}$, volunteered to participate in this study, giving written informed consent.

\section{Procedures}

An initial demographic interview was conducted. This was based on a common script that included a demographic questionnaire, medical history, drinking and drug habits and present health status. The MS data, such as type, duration, and degree of disability and severity, as assessed by the Expanded Disability Status Scale (EDSS) [22] score and the Multiple Severity Status Score (MSSS) [23], respectively, were obtained in the clinical protocols.

Then, participants underwent the BICAMS battery [14], which included the oral version of Symbol Digit Modalities Test (SDMT) [16], the learning trials from the California Verbal Learning Test-II (CVLT-II) [17] and the Brief Visuo-spatial Memory Test-Revised learning trials (BVMT-R) [18].

The SDMT [16] examines sustained attention, concentration and processing speed. In the oral version, the participant examines a series of nine meaningless geometric symbols, which are labeled from 1 to 9. Then, during $90 \mathrm{~s}$ the participant is instructed to say the corresponding number to each symbol, as rapidly as possible. The test score corresponds to the number of correct responses.

The CVLT-II [17] is a measure of verbal learning and memory. The test begins with the examiner reading a list of 16 words to the patient and then he/she is asked to report as many of the items as possible, in any order. After recall is recorded, the entire list is read again followed by a second attempt at recall. Altogether, there are five learning trials. The outcome measure is the total number of recalled items over the five learning trials.

The BVMT-R [18] is a measure of visuo-spatial learning and memory. The participant is exposed to a matrix of six simple abstract designs for $10 \mathrm{~s}$ followed by an unaided recall; we used the form 1 of the original test. After that, the participant is asked to render the designs using paper and pencil, taking as much time as needed for reproduction. The scoring criterion is based on location and accuracy of each design (from 0 to 2 , maximum total score for each array 12). The outcome measure of this test corresponds to the total recall score across the three trials.

The validation was conducted per the international standards given by the expert consensus committee [15]. As the first step, the CVLT-II list of words were translated and re-translated from English to Portuguese and vice versa respectively; the other two tests did not require translation due to their nature. In the second step, the test instructions were translated into Portuguese.

In both groups, anxiety and depression symptoms were also measured using the Portuguese version of Hospital Anxiety and Depression Scale (HADS) [24]. In the MS group the level of fatigue was measured with the Modified Fatigue Impact Scale (MFIS) [25-27].

"The participants of both groups were asked to return for a follow-up session to allow for test-retest reliability analyses. A subgroup of 26 patients and $13 \mathrm{HC}$ returned after a mean time of 7 months and all the tests administered in the first session were repeated in the same manner and in the same order." 
Well-trained clinical psychologists conducted all sessions and the tests were applied in a standardized way and in a fixed order. The mean time for BICAMS application was $15 \mathrm{~min}$, as described $[14,15]$.

\section{Statistical analysis}

Statistical analysis was performed using the Statistical Package for the Social Sciences (IBM SPSS), version 23.0. Descriptive statistics (e.g., mean, standard deviation, median, interquartile range and percentages) were used for demographic characterization of both groups. Student's t-test for independent samples was used to analyze the differences between groups, at the level of $p$ $<.05$. The values shown in the tables are bilateral $p$-values. The effect sizes of those differences were calculated using Cohen's $d$. Spearman's correlations (P) were used to analyze reliability measures and the relationship between BICAMS, HADS and MFIS results. Raw scores were analyzed for the full sample and Z-scores were calculated. Multiple regression analysis was used to produce normative data.

\section{Results}

\section{Demographics and MS characteristics}

The groups were similar with regard to age (MS group: $\mathrm{M}=38.26$ years $\pm 11.03 ; \mathrm{HC}: \mathrm{M}=36.17$ years \pm 12.01 , $p=.63$ ), gender (MS group: \%Female $=66.7$; HC: \%Female $=58.3, p=.28)$ or number of educational years (MS group: $\mathrm{M}=13.55 \pm 3.71 ; \mathrm{HC}: \mathrm{M}=14.62 \pm 3.47, p=.42$ ). With respect to professional status, the majority of subjects were employed, with a much higher proportion of HC than MS, as is usually reported $(n=56,94.9 \% ; n=$ $61,58.1 \%$, respectively). In the MS group, $92,4 \%(n=97)$ of patients had the relapsing remitting type and 3,8\% $(n=4)$ secondary progressive type and $3,8 \%(n=4)$ clinically isolated syndrome. The average disease duration was 6.52 years $(\mathrm{SD}=5.95)$ and the median EDSS score was 1.5 (range: 0-6.0). The MSSS score, calculated in patients from 1 to 30 years of disease duration $(n=95)$, had a median value of 2.01 (IQR range: 3.83 ).

\section{Criterion-related validity: Group differences}

Means, standard deviations and $t$ test's for independent samples from the three tests are presented in Table 1. The results showed that MS group performed significantly worse than the $\mathrm{HC}$ group on all measures.

Table 1 Group differences on BICAMS measures

\begin{tabular}{lllll}
\hline & $\begin{array}{l}\text { MS } \\
(N=105)\end{array}$ & $\begin{array}{l}\mathrm{HC} \\
(N=60)\end{array}$ & $t$ & $P$ \\
\hline SDMT & $51.77(11.20)$ & $58.68(10.02)$ & 3.77 & 0.000 \\
CVLT-II & $55.05(11.84)$ & $60.47(10.12)$ & 2.98 & 0.003 \\
BVMT-R & $21.72(7.27)$ & $24.68(5.52)$ & 2.94 & 0.004 \\
\hline
\end{tabular}

Cohen's $d$ was analyzed for each neuropsychological test and were satisfactory: SDMT - 0.65 (large); CVLT-II 0.49 (medium); BVMT-R - 0.45 (medium) [28].

\section{Reliability: Test-retest}

The test-retest reliability data obtained in a subgroup of MS patients are presented in Table 2. The test-retest reliability coefficients showed a strong to a very strong and significant effect for all BICAMS tests.

The test-retest results in the $\mathrm{HC}$ were not considered in view of the low number of cases.

\section{Regression based-norms}

To obtain a regression-based normative model for BICAMS, the distribution of the SDMT, CVLT-II and BVMT-R raw scores was analyzed for the complete sample and the $\mathrm{Z}$ scores were calculated. The raw scores were then converted into scaled scores $(M=10$ and $S D$ $=3$ ), as presented in Table 3. For each test a multiple regression analysis with a stepwise method using the scaled scores as dependent variable and age, gender and education as predictors was performed. Education was introduced as the number of regular academic school years that the participant successfully completed. As some studies suggest that there is a curvilinear relationship between demographic variables and cognitive function [29], the quadratic term of age and education were also introduced as predictors. These results allow us to detect which variables contributed significantly to explain each of the scaled neuropsychological test scores.

The T-scores corrected for education, age and gender were generated through a procedure suggested by Diehr and colleagues [30]. Therefore, another multiple regression (enter method) with each of the BICAMS test scaled scores as the dependent variable and the significant predictors of each test was performed. The non-standardized predicted values of this equation were saved and a new variable was calculated corresponding to the difference between an individual's actual and predicted scale score (i.e., the residual) divided by the standard deviation of those residuals. These values were then rescaled for a $\mathrm{T}$-score $(\mathrm{M}=50$ and $\mathrm{SD}=10)$.

Finally, another multiple regression analysis with corrected T-score as the dependent variable was performed to generate each test normative formula for the

Table 2 Test-retest means and correlations for MS group $(n=26)$

\begin{tabular}{|c|c|c|c|c|c|c|}
\hline & \multicolumn{2}{|l|}{ Time 1} & \multicolumn{2}{|c|}{ Time 2} & \multirow{2}{*}{$\begin{array}{l}\text { Spearman's } \\
\text { correlation }\end{array}$} & \multirow[t]{2}{*}{$P$} \\
\hline & Mean & $S D$ & Mean & $S D$ & & \\
\hline$\overline{\text { SDMT }}$ & 50.96 & 11.56 & 53.92 & 13.99 & 0.90 & $<0.001$ \\
\hline CVLT-II & 57.08 & 12.75 & 57.31 & 17.44 & 0.71 & $<0.001$ \\
\hline BVMT-R & 22.00 & 7.43 & 25.12 & 6.94 & 0.84 & $<0.001$ \\
\hline
\end{tabular}


Table 3 Raw score to scaled score conversions for the BICAMSs tests

\begin{tabular}{llll}
\hline Scaled score & SDMT & CVLT-II & BVMT-R \\
\hline 1 & - & $0-21$ & - \\
2 & $0-20$ & $22-27$ & $1-3$ \\
3 & $21-24$ & $28-33$ & $4-5$ \\
4 & $25-27$ & $34-37$ & $6-8$ \\
5 & $28-33$ & $38-41$ & $9-10$ \\
6 & $34-37$ & $42-44$ & $11-14$ \\
7 & $38-40$ & 45 & 15 \\
8 & $41-45$ & $46-49$ & $16-17$ \\
9 & $46-49$ & $50-53$ & $18-20$ \\
10 & $50-53$ & $54-57$ & $21-22$ \\
11 & $54-57$ & $58-60$ & $23-25$ \\
12 & $58-61$ & $61-64$ & $26-27$ \\
13 & $62-65$ & $65-68$ & $28-30$ \\
14 & $66-69$ & $69-72$ & $31-32$ \\
15 & $70-73$ & $73-76$ & $33-34$ \\
16 & $74-76$ & $77-79$ & - \\
17 & $77-78$ & - & - \\
\hline
\end{tabular}

Portuguese population. The final formula to calculate the T-scores for each of BICAMS's test are presented below:

$$
\begin{aligned}
\text { SDMT T score }= & 10.511+\left(0.007^{*} \text { age }^{2}\right) \\
+ & \left(-0.966^{*} \text { years of education }\right) \\
+ & \left(4.138^{*} \text { scaled score }\right) \\
\text { CVLT-II T score }= & 3.195+\left(0.006^{*} \text { age }^{2}\right) \\
& +\left(3.761^{*} \text { scaled score }\right) \\
\text { BVMT-R T score }= & -8.004+\left(0.514^{*} \text { age }\right) \\
& +\left(3,833^{*} \text { scaled score }\right)
\end{aligned}
$$

In determining impairment, the 5th percentile value based on the performance of healthy control sample was calculated for each test. Participants were considered impaired if their score was equal of below the percentile $5^{\text {th }}$ of the control group (results are presented on the Table 4) [31]. Then, using the previously reported criteria of impairment defined by "one or more abnormal

Table 4 The prevalence of cognitive impairment in MS patients according to the 5th percentile value of $\mathrm{HC}$ on BICAMS tests

\begin{tabular}{lll}
\hline & $\begin{array}{l}\text { 5th Percentile value for HC } \\
\text { on each test }\end{array}$ & $\begin{array}{l}\text { Percentage of MS patients under } \\
\text { 5th percentile }\end{array}$ \\
\hline SDMT & 38 & $14.3 \%$ \\
CVLT-II & 41 & $9.5 \%$ \\
BVMT-R & 12 & $11.4 \%$ \\
\hline
\end{tabular}

tests" [32, 33], it was found that $24.8 \%$ of the MS sample was impaired at baseline.

Analysing the degree of disability assessed by EDSS and cognitive performance, we found significant correlations with all cognitive tests (SDMT: $-.497, p=.000$; CVLT: $-.334, \mathrm{p}=.000$; BVMT: $-.275, p=.005)$.

Regarding anxiety and depression symptoms, it was found that MS group presented higher scores on these measures than $\mathrm{HC}$, and that these differences were statistically significant: anxiety (MS group: $\mathrm{M}=7.85 \pm 4.51$; $\mathrm{HC}$ : $\mathrm{M}=6.32 \pm 3.00, t=-2.348 ; p=.20$ ) and depression (MS group: $\mathrm{M}=5.14 \pm 3.95 ; \quad \mathrm{HC}: \mathrm{M}=3.18 \pm 2.57$ ). Anxiety symptoms were found to be more frequent $(n=56 ; 53.3 \%)$ than depression symptoms $(n=29 ; 27.6 \%)$ in MS patients. In the MS group depression symptoms had a modest significantly negative effect only on CVLT-II results ( $\mathrm{R}$ 0.196; $p=.45$ ), whereas anxiety was not significantly correlated with any BICAMS test. The assessment with the MFIS scale $(n=95)$ showed that fatigue was present in 50 MS patients $(47,4 \%)$ and was significantly correlated with the EDSS score $(\mathrm{R}-.279 ; p=.006)$, and with anxiety $(\mathrm{R}-$ $.631 ; p=.0001)$ and depression symptoms ( $\mathrm{R}-.754 ; p$ $=.0001)$. Conversely, fatigue was negatively correlated with SDMT score $(\mathrm{R}-.266 ; p=.009)$; similar results were observed in both MFIS subscales, physical $(\mathrm{M}=18.04 \pm$ 9.66; R-.289; $p=.005)$ and cognitive $(M=17.84 \pm 10.21$; $\mathrm{R}-.203 ; p=.049)$.

\section{Discussion}

An international consensus committee of experts recently recommended a short battery of tests for cognitive assessment in MS that allows monitoring of cognition over time and is a fast and reliable instrument that may be administered by healthcare professionals with no specific experience in neuropsychological testing. According to the international standards for validation [15], several validation studies of BICAMS have been carried out in different cultures and languages, with the aim of making this psychometric tool more solid and internationally applicable. Up to now, there exists normative data for populations of several countries, such as Czech Republic [32], Italy [34], Hungary [35], Ireland [36], Brazil [37], Lithuania [38] Argentina [39], Canada [33], Greece [31], Belgium [40], Japan [41] and Turkish [42].

The current study followed the recommendations and standards of the BICAMS consensus committee $[14,15]$ and is the first to publish the Portuguese normative data for SDMT, CVLT-II and BVMT-R. Our results showed that MS group performed significantly worse than $\mathrm{HC}$ group on all measures (SDMT, CVLT-II and BVMT-R), a finding that is in agreement with the other recently published validations. These differences were more marked in the SDMT and CVLT-II than the BVMT-R, 
and similar results were found by O'Connell and colleagues (2015), Spedo and colleagues (2015) and Vanotti and colleagues (2016). Test-retest reliability in our population fits the recommended international standards for BICAMS validation [14]. Test-retest reliability for raw scores was adequate to excellent for all the three tests in this validation; more than 80 in SDMT and BVMT, replicating prior finds $[33,34]$. Yet our results are lower than those of Vanotti and colleagues (2016). In addition we confirmed that the SDMT has particularly high test-retest reliability. We used a wider time span than other authors $[37,40]$ in order to avoid the learning effect, since at both evaluation times the same forms were applied.

The BICAMS tasks were able to identify cognitive impairment in $24.8 \%$ of MS patients using the criteria of impairment defined by one or more abnormal tests. This is a lower value than those found in other studies, which ranged from 47.3 to $58 \%$ [31-33, 35, 36]. This result may reflect the characteristics of our MS sample, which were mainly RRMS and rather early cases (mean disease duration 6.5 years) and a correspondingly low level of physical disability, median EDSS 1.5 [31-33, 36]. The lower level of disability in our sample is further supported by our MSSS data [23].

We found a significant correlation between EDSS and cognitive performance in the three tests used, that is, the higher the EDSS score the worse the cognitive test performance.

Regarding anxiety and depression symptoms, we found that the MS group also presented with higher scores on these measures then the $\mathrm{HC}$, fitting the results of other BICAMS validation studies [32, 33, 37]. The Hungarian BICAMS validation reported a negative correlation of fatigue with all BICAMS tests [31]. In our study an association with fatigue was only seen in the SDMT test, possibly reflecting the lower fatigue in our patients as well as the lower physical disability.

This study was some limitations. First, follow-up assessments were done in a low number of cases, especially in the $\mathrm{HC}$ group, which is due to the fact that some individuals live far from the Hospital and incur additional personal costs. Another limitation is the fact that effect size for CVLT and BVMT-R although satisfactory, is on the threshold of the effect size classified as medium.

\section{Conclusions}

In conclusion, our study provides the Portuguese BICAMS standards for use with MS patients and evidences the strong psychometric properties of the Portuguese BICAMS version. The normative data of the BICAMS for the Portuguese population enables the use of the battery in clinical practice, for longitudinal patient assessments and as an outcome measure of cognitive functioning in clinical trials. Future prospective studies with larger samples of MS patients, with different types of disease evolution, will certainly add valuable information concerning the clinical applicability of the Portuguese BICAMS version.

\section{Abbreviations}

BICAMS: Brief International cognitive assessment for multiple sclerosis; BRB$\mathrm{N}$ : Brief repeatable battery of neuropsychological tests; BVMT-R: Brief visuospatial memory test - revised; Cl: Cognitive impairment; CVLT-II: California verbal learning test - II; EDSS: Expanded disability status scale; HADS: Hospital anxiety and depression scale; HC: Healthy subjects; IBM SPSS: Statistical package for the social sciences; MACFIMS: Minimal assessment of cognitive function in multiple sclerosis; MFIS: Modified fatigue impact scale; MoCa: Montreal cognitive assessment; MS: Multiple sclerosis; MSSS: Multiple severity status score; SDMT: Symbol digit modalities test

\section{Acknowledgements}

The authors would like to thank all participants of this study. An earlier version of this paper was presented at the ECTRIMS-ACTRIMS Meeting, Paris, France, 25-28 October 2017. The abstract of the e-Poster was published in the Multiple Sclerosis Journal (2017): 23: (S3): 680 - 975. http://journals.sagepub.com/doi/10.1177/1352458517731285.

\section{Availability of data materials}

The datasets used and analysed during the current study are available from the corresponding author on reasonable request.

\section{Funding}

This work was funded by an unrestricted educational grant from Bayer, which had any role in the study, namely in its design, sample collection, analyses and interpretation of data and in the writing of the manuscript.

\section{Authors' contributions}

CS contributed in study concept and design, drafting and revising the manuscript and in the acquisition and interpretation of data. MRN contributed in study concept and design, drafting and revising the manuscript, in the acquisition and interpretation of data and statistical analysis. TM contributed in the acquisition of data and statistical analysis. PA and JV contributed in patient recruitment, acquisition of clinical data and revising the manuscript. AMP contributed in study concept and design, revising the manuscript, in the interpretation of data and statistical analysis. DL contributed in study concept and design and in revising the manuscript. MJS contributed in study concept and design, drafting and revising the manuscript, in the analysis and interpretation of data and study supervision. All authors read and approved the final manuscript.

\section{Ethics approval and consent to participate}

All patients and all healthy control subjects provided written informed consent to participation in the study. The Ethics Committees of Centro Hospital S. João, Oporto and Ethics Committees Hospital Beatriz Ângelo, Loures, granted approval for the research project.

\section{Consent for publication}

Not applicable.

\section{Competing interests}

MJS has received consulting/speaker fees from Bayer, Biogen, CSL Behring, Merck, Novartis, Roche, Sanofi and Teva.

DL has participated in speaker bureau for Bayer, Merck, Almirall, Execemed, TEVA, Roche, Novartis, Biogen, Sanofi; has had consultancy from Novartis, Bayer, Merck, Biogen, TEVA, Sanofi; has had research grants from Bayer, Merck, Novartis, Biogen. All are paid into DL's institution.

The other authors have nothing to disclose regarding this study.

\section{Publisher's Note}

Springer Nature remains neutral with regard to jurisdictional claims in published maps and institutional affiliations. 


\section{Author details}

'MS Clinic, Department of Neurology, Centro Hospitalar São João Porto, Alameda Prof. Hernâni Monteiro, 4200 - 319 Porto, Portugal. ${ }^{2}$ BRU-IUL, Instituto Universitário de Lisboa (ISCTE-IUL), Lisbon, Portugal. ${ }^{3}$ Department of Neurology, Hospital Beatriz Ângelo, Loures, Portugal. ${ }^{4}$ Department of Psychology, Royal Holloway, University of London, London, UK. ${ }^{5}$ Faculty of Health Sciences, University Fernando Pessoa, Porto, Portugal.

\section{Received: 8 May 2018 Accepted: 7 October 2018}

\section{Published online: 17 October 2018}

\section{References}

1. Rao SM, Leo GJ, Bernardin L, Unverzagt F. Cognitive impairment in multiple sclerosis. I. Frequency, patterns, and prediction. Neurology. 1991;41:685-91.

2. Amato MP, Zipoli V, Portaccio E. Multiple sclerosis-related cognitive changes: a review of cross-sectional and longitudinal studies. J Neurol Sci. 2006;245:1-2.

3. Langdon DW. Cognition in multiple sclerosis. Curr Opin Neurol. 2011;24(3): 244-9.

4. Glanz BI, Holand M, Gauthier SA, Amunwa EL, Liptak Z, Houtchens MK Sperling RA, Khoury SJ, Guttmann CR, Weiner HL. Cognitive dysfunction in patients with clinically isolated syndromes or newly diagnosed multiple sclerosis. Mult Scler. 2007;13(8):1004-10

5. Amato MP, Hakiki B, Goretti B, et al. Association of MRI metrics and cognitive impairment in radiologically isolated syndromes. Neurology. 2012; 78:309-14.

6. Zipoli V, Goretti B, Hakiki B, et al. Cognitive impairment predicts conversion to multiple sclerosis in clinically isolated syndromes. Mult Scler. 2010;16:62-7.

7. Kobelt G, Thompson A, Berg J, et al. New insights into the burden and costs of multiple sclerosis in Europe. Mult Scler. 2017;23(8):1123-36.

8. Sá MJ, Kobelt G, Berg J, Capsa D, Dalén J. European Multiple Sclerosis Platform. New insights into the burden and costs of multiple sclerosis in Europe: Results for Portugal. Mult Scler. 2017;23(2-suppl):143-54.

9. Langdon D. Cognitive assessment in MS. Neurodegenerative Disease Management. 2015;5(6s):43-5.

10. Rimkus MC, Steenwijk MD, Barkhof F. Causes, effects and connectivity changes in MS-related cognitive decline. Dement Neuropsychology. 2006; 10(1):2-11.

11. Vanotti S, Caceres FJ. Cognitive and neuropsychiatric disorders among MS patients from Latin America. Multiple Sclerosis: Journal experimental translational clinical. 2017:1-11.

12. Giedraitiene N, Kaubrys G, Kizlaitiene R. Cognition during and after multiple sclerosis relapse as assesses with the brief international cognitive assessment for multiple sclerosis. Sci Rep. 2018:8:8169.

13. Benedict RH, Cookfair D, Gavett R, Gunther M, Munschauer F, Garg N, Weinstock-Guttman B. Validity of the minimal assessment of cognitive function in multiple sclerosis (MACFIMS). J Int Neuropsychol Soc. 2006;12:549-58.

14. Langdon DW, Amato MP, Boringa J, Brochet B, Foley F, Fredrikson S, Hämäläinen P, Hartung HP, Krupp L, Penner IK, Reder AT, Benedict RH. Recommendations for a brief international cognitive assessment for multiple sclerosis (BICAMS). Mult Scler. 2012;18:891-8.

15. Benedict RH, Amato MP, Boringa J, Brochet B, Foley F, Fredrikson S, Hamalainen P, Hartung H, Krupp L, Penner I, Reder AT, Langdon DW. Brief international cognitive assessment for MS (BICAMS): international standard for validation. BMC Neurol. 2012:16:55.

16. Smith A. Symbol digit modalities test: manual. Los Angeles, CA: Western Psychological Services; 1982.

17. Delis DC, Kramer JH, Kaplan E, Ober BA. California verbal learning test, second edition (CVLT-II). San Antonio, TX: Psychological Corporation; 2000.

18. Benedict RHB. The brief visuospatial memory test revised (BVMT-R). Lutz, FL: Psychosocial Assessment Resources Inc.; 1997.

19. Polman CH, Reingold SC, Banwell B, Clanet M, Cohen JA, Filippi M, Wolinsky JS. Diagnostic criteria for multiple sclerosis: 2010 revisions to the McDonald criteria. Ann Neurol. 2011;69(2):292-302.

20. Nasreddine ZS, Phllips NA, Béridian V, Charbonneau S, Whitehead V, Collin I, Cummings $\mathrm{L}$, Chertkow $\mathrm{H}$. The Montreal cognitive assessment, MoCA: a brief screening tool for mild cognitive impairment. J Am Geriatr Soc. 2005; 53(4):695-9.

21. Freitas $S$, Simões MR, Alves L, Santana I. Montreal cognitive assessment (MoCA): normative study for the Portuguese population. J Clin Exp Neuropsychol. 2011;33(9):989-96.
22. Kurzke JF. Rating neurologic impairment in multiple sclerosis: an expanded disability status scale (EDSS). Neurology. 1983;33:1444-52.

23. Roxburgh RHSR, Seaman SR, Masterman T, Hensiek AE, et al. Multiple sclerosis severity score using disability and disease duration to rate disease severity. Neurology. 2005;64:1144-51.

24. Silva AM, Vilhena E, Lopes A, Santos E, Gonçalves MA, Pinto C, et al. Depression and anxiety in a Portuguese MS population: association with physical disability and severity of disease. J Neurol Sci. 2011;306:66-70.

25. Fisk JD, Ritvo PG, Haase DA, Marrie TJ, Schlech WF. Measuring the functiona impact of fatigue: initial validation of the fatigue impact scale. Clin Infect Dis. 1994;18(Suppl 1):S79-83.

26. Larson RD. Psychometric properties of the modified fatigue impact scale. Int J MS Care. 2013:15(1):15-20.

27. Gomes LR. Validação da versão portuguesa da Escala de Impacto da Fadiga Modificada e da Escala de Severidade da Fadiga na Esclerose Múltipla (Validation of the Portuguese version of the Modified Fatigue Impact Scale and the Fatigue Severity Scale in Multiple Sclerosis). Thesis.Minho University. 2011

28. Cohen J. Statistical power analysis for the behavioral sciences. New York, NY: Routledge Academic; 1988

29. Strauss E, Sherman E, Spreen O. A compendium of neuropsychological tests: administration, norms and commentary. 3rd ed. Oxford, UK: University Oxford Press; 2006.

30. Diehr MC, Cherner M, Wolfson TJ, Miller SW, Grant I, Heaton RK, the HIV Neurobehavioral Research Center. The 50 and 100-item short forms of the paced auditory serial addition task (PASAT): demographic corrected norms and comparisons with the full PASAT in normal and clinical samples. J Clin Exp Neuropsychol. 2003;25:571-58.

31. Polychroniadou E, Bakirtzis C, Langdon D, Lagoudaki E, Kesidou E, Theotokis $P$, et al. Validation of the brief international cognitive assessment for multiple sclerosis (BICAMS) in Greek population with multiple sclerosis. Multiple Sclerosis and Related Disorders. 2016:9:68-72.

32. Dusankova JB, Kalincik T, Havrdova E, Benedict RH. Cross cultural validation of the minimal assessment of cognitive function in multiple sclerosis (MACFIMS) and the brief international cognitive assessment for multiple sclerosis (BICAMS). Clin Neuropsychol. 2012;26(7):1186-200.

33. Walker LA, Osman L, Berard JA, Rees LM, Freedman MS, MacLean $H$, Cousineau D. Brief international cognitive assessment for multiple sclerosis (BICAMS): Canadian contribution to the international validation project. J Neurol Sci. 2006:362:147-52.

34. Goretti B, Niccolai C, Hakiki B, Sturchio A, Falautano M, Minacapelli E, Amato $M$. The brief international cognitive assessment for multiple sclerosis (BICAMS): normative values with gender, age and education corrections in the Italian population. BMC Neurol. 2014;14:171

35. Sandi D, Rudisch T, Füvesi J, Fricska-Nagy Z, Huszka H, Biernacki T, Bencsik K The Hungarian validation of the brief international cognitive assessment for multiple sclerosis (BICAMS) battery and the correlation of cognitive impairment with fatigue and quality of life. Multiple Sclerosis and Related Disorders. 2015:4:499-504

36. O'Connell K, Langdon D, Tubridy N, Hutchinson M, McGuigan C. A preliminary validation of the brief international cognitive assessment for multiple sclerosis (BICAMS) tool in an Irish population with multiple sclerosis (MS). Multiple Sclerosis and Related Disorders. 2015: 4:521-5.

37. Spedo CT, Frndak SE, Marques VD, Foss MP, Pereira DA, Carvalho L, Barreira AA. Cross-cultural adaptation, reliability, and validity of the BICAMS in Brazil. Clin Neuropsychol. 2015;29:836-46.

38. Giedraitienè N, Kizlaitienè R, Kaubrys G. The BICAMS battery for assessment of Lithuanian-speaking multiple sclerosis patients: relationship with age, education, disease disability, and duration. Med Sci Monit. 2015:21:3853-9.

39. Vanotti S, Smerbeck A, Benedict RHB, Caceres F. A new assessment tool for patients with multiple sclerosis from Spanish-speaking countries: validation of the Brief International Cognitive Assessment for MS (BICAMS) in Argentina. Neurology. 2015:84(14):Suppl.P5. 201

40. Costers L, Gielen J, Eelen PL, Schependom JV, Laton J, Remoortel AV, Vanzeir E, Wijmeersch BV, Seeldrayers P. Haelewyck MC, D'Haeseleer M, D'hooghe MB, Langdon D, Nagels G. Does including the full CVLT-II and BVMT-R improve BICAMS? Evidence from a Belgian (Dutch) validation study. Multiple Sclerosis Related Disorders. 2017;18:33-40.

41. Niino M, Fukazawa T, Kira Il, Okuno T, Mori M, Sanjo N, Ohashi T, Fukaura H, Fujimori J, Shimizu Y, Mifune N, Miyazaki Y, Takahashi E, Kikuchi S, Langdon 
$\mathrm{D}, \mathrm{Hb}$ Benedict $\mathrm{R}$, Matsui M. Validation of the brief international cognitive assessment for multiple sclerosis in Japan. Mult Scler J Exp Transl Clin. 2017; 26, 3(4).

42. Ozakbas S, Yigit P, Cinar BP, Limoncu H, Kahraman T, Kösehasanoğulları G.

The Turkish validation of the brief international cognitive assessment for multiple sclerosis (BICAMS) battery. BMC Neurol. 2017;17:208.

Ready to submit your research? Choose BMC and benefit from:

- fast, convenient online submission

- thorough peer review by experienced researchers in your field

- rapid publication on acceptance

- support for research data, including large and complex data types

- gold Open Access which fosters wider collaboration and increased citations

- maximum visibility for your research: over $100 \mathrm{M}$ website views per year

At $B M C$, research is always in progress.

Learn more biomedcentral.com/submissions 